\title{
ON CLOSED OPERATORS WITH CLOSED RANGE ${ }^{1}$
}

\section{J. T. JOICHI}

It is well-known that if $T$ is an everywhere defined bounded operator on a Banach space $X$ to a Banach space $Y$ and $T^{*}$ is its adjoint, then the range $R\left(T^{*}\right)$ of $T^{*}$ is closed in $X^{*}$ if and only if the range $R(T)$ of $T$ is closed in $Y$ (cf. [2, pp. 487-489]). The object of this note is to establish this result for closed but possibly unbounded operators. This result, for the unbounded case, is of great utility in the study of differential operators and has been considered by $\mathrm{F}$. E. Browder, ${ }^{2}$ I. C. Gohberg and M. G. Krein, and by G. C. Rota.

In the sequel, we shall have occasion to consider a set under several different topologies. We shall use the following convention: if $A$ is a linear set and $B$ is a set of linear functionals on $A$, then the set $A$ with the weak topology induced by the elements of $B$ will be denoted by $(A, B)$. Thus, the assertion that a set $C$ is closed (dense, etc.) in ( $A, B$ ) shall mean that $C$ is a subset of $A$ which is closed (dense, etc.) in this weak topology. If $A$ is a Banach space, then the assertion that a set $C$ is closed (dense, etc.) in $A$ shall refer to the norm topology of $A$.

Henceforth, $X$ and $Y$ will be Banach spaces. If $T$ is a closed operator with domain $D(T)$ in $X$ and range $R(T)$ in $Y$, then it was noted by Sz.-Nagy [5], that $D(T)$ becomes a Banach space under the norm $|x|_{T}=|x|+|T x|$ (which we shall call the $T$-norm) and $T$ is a bounded operator on this space. If in addition, $D(T)$ is dense in $X$, it is well known that $T$ has a uniquely defined adjoint $T^{*}$ with domain $D\left(T^{*}\right)$ dense in $\left(Y^{*}, Y\right)$, and that $T^{*}$ is a closed operator.

Theorem $1 .^{3}$ Let $T$ be a closed operator on $X$ to $Y$ with $D(T)$ dense in $X$. Then the range $R\left(T^{*}\right)$ is closed in $X^{*}$ if and only if the range $R(T)$ is closed in $Y$.

That $R\left(T^{*}\right)$ is closed in $X^{*}$ when $R(T)$ is closed in $Y$ is shown in

Received by the editors April 3, 1959.

1 This note was written under grant G-7011 of the National Science Foundation.

${ }^{2}$ It has been learned through personal communication that Browder has established this and related results in a forthcoming paper [1]. However, as he deals with more general spaces, his proof requires considerably more analysis.

${ }^{3}$ Since submitting this paper, it was discovered that T. Kato has a proof of this result in print (Perturbation theory for nullity, deficiency and other quantities of linear operators, J. Analyse Math. vol. 6 (1958) p. 273). However, his method of proof is completely different from that contained herein. 
$[3$, p. 98] to follow from the bounded case after introducing the $T$ norm on $D(T)$. Alternatively, a straight-forward modification of the proof for the bounded case can be used. The converse is shown to be true in [3, p. 99] but only under the restriction that $X$ and $Y$ be reflexive. Rota [4] shows that the converse for general Banach spaces follows easily from Theorem 2 below, but there is one assertion in his proof of this latter result which does not hold in general. However, with the aid of the following lemmas, a modification of his argument can be used.

Lemma 1. Let $T$ be a closed operator on $X$ to $Y$ with $D(T)$ dense in $X$. Then $T^{*}$ is continuous (and hence uniformly continuous) as an operator with domain $\left(D\left(T^{*}\right), Y\right)$ and range in $\left(X^{*}, D(T)\right)$.

Proof. A basic neighborhood of 0 in $\left(X^{*}, D(T)\right)$ is given by

$$
N=\left\{x^{*} \in X^{*}:\left|x^{*}\left(x_{i}\right)\right|<\epsilon, i=1, \cdots, n\right\},
$$

where each $x_{i}$ is in $D(T)$. Consider the following neighborhood of 0 in $\left(D\left(T^{*}\right), Y\right)$ :

$$
M=\left\{y^{*} \in D\left(T^{*}\right):\left|y^{*}\left(T x_{i}\right)\right|<\epsilon, i=1, \cdots, n\right\} .
$$

Clearly, $T^{*}(M) \subset N$. (This is a reinterpretation of part (F) of Rota's proof of Theorem 2 (cf. [4, p. 28]).)

Since $T^{*}$ is a closed operator, the set $D\left(T^{*}\right)$ is a Banach space under the $T^{*}$-norm. Let $E$ be this Banach space and let $S_{E}$ be its unit ball.

Lemma 2. Let $T$ be a closed operator on $X$ to $Y$ with $D(T)$ dense in $X$. Then $S_{E}$ is a compact subset of $\left(Y^{*}, Y\right)$.

Proof. Let $A$ be a directed set and let $\left\{y_{a}^{*}: a \in A\right\} \subset S_{E}$ be a Cauchy net in $\left(Y^{*}, Y\right)$. Let $S_{Y}$ be the unit ball of $Y^{*}$ in the usual norm. Then, by the Banach-Alaoglu Theorem [2, p. 424], $S_{Y}$ is compact in $\left(Y^{*}, Y\right)$. Since for every $y^{*}$ in $D\left(T^{*}\right),\left|y^{*}\right| T^{*} \geqq\left|y^{*}\right|$, we have $S_{E} \subset S_{Y}$ and hence there exists a $y_{0}^{*}$ in $S_{Y}$ such that $y_{a}^{*} \rightarrow y_{0}^{*}$ in $\left(Y^{*}, Y\right)$. By Lemma $1,\left\{T^{*}\left(y_{a}^{*}\right): a \in A\right\}$ is a Cauchy net in $\left(X^{*}, D(T)\right)$. By the boundedness of $T^{*}$ as an operator on $E$ to $X^{*}, T^{*}\left(S_{E}\right) \subset n S_{X}$ for some integer $n>0$, where $S_{X}$ is the unit ball of $X^{*}$ in the usual norm. Now, the topology of $\left(X^{*}, D(T)\right)$ is weaker than the topology of $\left(X^{*}, X\right)$, and hence $n S_{X}$ is compact in $\left(X^{*}, D(T)\right)$. Thus, there exists an $x_{0}^{*}$ in $n S_{X}$ such that $T^{*}\left(y_{a}^{*}\right) \rightarrow x_{0}^{*}$ in $\left(X^{*}, D(T)\right)$, i.e., for all $x$ in $D(T), T^{*} y_{a}^{*}(x) \rightarrow x_{0}^{*}(x)$. But for $x$ in $D(T), T^{*} y_{a}^{*}(x)=y_{a}^{*}(T x)$ $\rightarrow y_{0}^{*}(T x)$. Hence, for all $x$ in $D(T), y_{0}^{*}(T x)=x_{0}^{*}(x)$. But then, by the definition of $T^{*}, y_{0}^{*}$ is in $D\left(T^{*}\right)$ and $T^{*}\left(y_{0}^{*}\right)=x_{0}^{*}$. For all $y$ in $Y$ we 
have $\left|y_{0}^{*}(y)\right|=\lim \left|y_{a}^{*}(y)\right| \leqq \lim \inf \left|y_{a}^{*}\right||y|$, and so $\left|y_{0}^{*}\right|$ $\leqq \lim$ inf $\left|y_{a}^{*}\right|$. Similarly, since $\left|x_{0}^{*}(x)\right| \leqq \lim$ inf $\left|T^{*} y_{a}^{*}\right||x|$ for $x$ in $D(T)$ and $D(T)$ is dense in $X$, we have $\left|x_{0}^{*}\right| \leqq \lim$ inf $\left|T^{*} y_{a}^{*}\right|$. Hence

$$
\begin{aligned}
\left|y_{0}^{*}\right| T^{*} & =\left|y_{0}^{*}\right|+\left|x_{0}^{*}\right| \\
& \leqq \liminf _{A}\left[\left|y_{a}^{*}\right|+\left|T^{*} y_{a}^{*}\right|\right]=\liminf _{A}\left|y_{a}^{*}\right| T^{*} .
\end{aligned}
$$

Since each $y_{a}^{*}$ is in $S_{E}$, we get $\left|y_{0}^{*}\right| T^{*} \leqq 1$, i.e., $y_{0}^{*}$ is in $S_{E}$. This proves that $S_{E}$ is complete in $\left(Y^{*}, Y\right)$. But $S_{E} \subset S_{Y}$ and $S_{Y}$ is compact in $\left(Y^{*}, Y\right)$, hence $S_{E}$ is compact in $\left(Y^{*}, Y\right)$.

Lemma 3. Suppose that $T$ is a closed operator on $X$ to $Y$ with $D(T)$ dense in $X$ and such that $T^{*}$ is one-to-one and $R\left(T^{*}\right)$ is closed. If $B$ is a closed convex subset of $\left(Y^{*}, Y\right)$, then $T^{*}(B)$ is closed in $\left(X^{*}, X\right)$.

Proof. To show that $T^{*}(B)$ is closed in $\left(X^{*}, X\right)$, it suffices, by the Krel̆n-Šmul'yan Theorem [2, p. 429], to show that $T^{*}(B) \cap m S_{X}$ is closed in $\left(X^{*}, X\right)$ for every $m>0$, where $S_{X}$ is the unit ball of $X^{*}$ in the usual norm. Since the set $m S_{X}$ under the topologies induced from either $\left(X^{*}, X\right)$ or $\left(X^{*}, D(T)\right)$, is a compact Hausdorff space, the two topologies are equivalent on $m S_{X}$. Thus, it suffices to show that $T^{*}(B) \cap m S_{X}$ is closed in $\left(X^{*}, D(T)\right)$. Since $m S_{X}$ is closed in $\left(X^{*}, D(T)\right)$, the set $T^{*-1}\left(m S_{X}\right)$ is closed in $\left(D\left(T^{*}\right), Y\right)$ by Lemma 1 . But $\left(D\left(T^{*}\right), Y\right)$ is just the set $D\left(T^{*}\right)$ with the topology of $\left(Y^{*}, Y\right)$ relativised to $D\left(T^{*}\right)$. Hence there exists a closed subset $C$ of $\left(Y^{*}, Y\right)$ such that $T^{*-1}\left(m S_{X}\right)=D\left(T^{*}\right) \cap C$. Now, $T^{*}$ is continuous on $E$ to $X^{*}$ and by hypothesis, is one-to-one and has closed range. Thus, by the Interior Mapping Principle [2, p. 55], there exists a $k>0$ such that $T^{*-1}\left(m S_{X}\right) \subset k S_{E}$, where $k S_{E}$ is compact in $\left(Y^{*}, Y\right)$ by Lemma 2 . Therefore, $T^{*-1}\left(m S_{X}\right)=k S_{E} \cap C$ is compact in $\left(D\left(T^{*}\right), Y\right)$. Hence, $B \cap T^{*-1}\left(m S_{X}\right)$ is compact in $\left(D\left(T^{*}\right), Y\right)$. By Lemma 1 it follows that $T^{*}(B) \cap m S_{X}=T^{*}\left(B \cap T^{*-1}\left(m S_{X}\right)\right)$ is compact and hence closed in $\left(X^{*}, D(T)\right)$. This establishes the lemma.

In $[4$, p. $28,(\mathrm{E})]$, Rota asserts that $S_{E}$ is compact in $\left(E, E^{*}\right)$. To see that this need not be the case, let $T$ be a bounded operator and $Y$ a nonreflexive Banach space. Then the norms on $E$ and $X^{*}$ are equivalent and $E^{*}=X^{* *}$. Thus, the compactness of $S_{E}$ in $\left(E, E^{*}\right)$ would imply the reflexivity of $Y$.

Theorem 2. Suppose that $T$ is a closed operator on $X$ to $Y$ with $D(T)$ dense in $X$ and such that $T^{*}$ is one-to-one and $R\left(T^{*}\right)$ is closed. Then $T$ maps $D(T)$ onto all of $Y$.

Proof. We give a brief sketch of parts (D) and $(\mathrm{H})$ of $[4, \mathrm{p} .28]$. 
Take $y_{0}$ in $Y, y_{0} \neq 0$, and let $B=\left\{y_{0}\right\}^{\perp}$, the annihilator in $Y^{*}$ of $y_{0}$. Then $B$ is a closed convex subset of $\left(Y^{*}, Y\right)$ and hence, by Lemma 3 , $T^{*}(B)$ is closed in $\left(X^{*}, X\right)$. By the totality of $D\left(T^{*}\right)$ over $Y$, i.e., the density of $D\left(T^{*}\right)$ in $\left(Y^{*}, Y\right)$, there exists a $y_{0}^{*}$ in $D\left(T^{*}\right)$ such that $y_{0}^{*}\left(y_{0}\right)=1$. Since $T^{*}$ is one-to-one, $T^{*}\left(y_{0}^{*}\right)$ does not belong to the set $T^{*}(B)$. Hence [2, p. 422], there exists $x_{0}$ in $X$ such that $\left(T^{*} y_{0}^{*}\right)\left(x_{0}\right)=1$ and $x^{*}\left(x_{0}\right)=0$ for all $x^{*}$ in $T^{*}(B)$. It is then shown that $x_{0}$ is in $D(T)$ and $T x_{0}=y_{0}$, completing the proof of the present theorem.

Theorem 1 can be proved from Theorem 2 as in [4, p. 29]. We now give an alternate proof. Let $Y_{1}$ be the closure of $R(T)$ in $Y$ and let $T_{1}$ be the operator on $X$ to $Y_{1}$ defined by $T_{1}(x)=T(x)$ for $x$ in $D(T)$. Also, let $\hat{T}^{*}$ denote the mapping on $Y^{*} / Y_{1}^{\perp}$ to $X^{*}$ which is induced by $T^{*}$. Now, since $Y_{1}^{*}=Y^{*} / Y_{1}^{\perp}$, it follows that $T_{1}^{*}=\hat{T}^{*}$ and hence $R\left(T_{1}^{*}\right)=R\left(\hat{T}^{*}\right)=R\left(T^{*}\right)$ is closed in $X^{*}$. Since $T_{1}^{*}$ is also one-to-one, we infer from Theorem 2 that $R(T)=R\left(T_{1}\right)$ is closed in $Y_{1}$ and hence in $Y$, which establishes the theorem.

\section{REFERENCES}

1. F. E. Browder, Functional analysis and partial differential equations, I, Math. Ann. vol. 138 (1959) pp. 55-79.

2. N. Dunford and J. T. Schwartz, Linear operators, vol. 1, New York, Interscience, 1958.

3. I. C. Gohberg and M. G. KreIn, The basic propositions on defect numbers, root numbers and indices of linear operators, Uspehi Mat. Nauk vol. 12 (1957) pp. 43-118 (Russian).

4. G. C. Rota, Extension theory of differential operators, I, Comm. Pure Appl. Math. vol. 11 (1958) pp. 23-65.

5. B. Sz.-Nagy, On the stability of the index of unbounded linear transformations, Acta Math. Acad. Sci. Hungar. vol. 3 (1952) pp. 49-52.

UNIVERSITY OF ILLINOIS 